\title{
The tumor microenvironment promotes cancer progression and cell migration
}

\author{
Viviana Salvatore ${ }^{1}$, Gabriella Teti $^{1}$, Stefano Focaroli ${ }^{1}$, Maria Carla Mazzotti ${ }^{2}$, \\ Antonio Mazzotti ${ }^{3}$, Mirella Falconi ${ }^{1}$ \\ ${ }^{1}$ DIBINEM, University of Bologna Department of Biomedical and Neuromotor Sciences, 40126 Bologna, Italy \\ ${ }^{2}$ DIMEC, University of Bologna, Department of Medical and Surgical Sciences, 40126 Bologna, Italy \\ ${ }^{3}$ Rizzoli Orthopaedhic Institute, 40136 Bologna, Italy \\ Correspondence to: Mirella Falconi, email: mirella.falconi@unibo.it \\ Keywords: tumor microenvironment, osteosarcoma, human fibroblasts, co-culture, tumor stroma \\ Received: July 14, $2016 \quad$ Accepted: November 04, $2016 \quad$ Published: December 24, 2016
}

ABSTRACT

The tumor microenvironment contributes to cancer progression, in part through interactions between tumor and normal stromal cells. This study analyzed morphological and molecular changes induced in co-cultured human fibroblasts (HFs) and the MG-63 osteosarcoma cell line. Co-cultured cell monolayers were morphologically analyzed using high resolution scanning electron microscopy (HR-SEM), and trans-well assays were performed to assess cell migration and invasion. Proteins involved in inflammatory responses, cancer cell invasion, and angiogenesis were assessed using western blotting. HR-SEM showed progressive spatial orientation loss by fibroblasts in contact with MG-63s, while MG-63s proliferated rapidly and invaded HF space. Trans-well assays showed enhanced MG-63 migration in the presence of HFs. IL- 6 expression was increased in co-cultured HFs, possibly stimulated by TNF-a. HFs do not normally express YKL-40 but did so in co-culture. Band densitometric analyses showed that increasing YKL-40 expression was followed by VEGF overexpression, especially in MG-63s. Finally, our results confirmed fibroblasts as the main matrix metalloproteinase source in this tumor microenvironment. Our study sheds new light on how tumor-stroma interactions promote tumor development and progression, and may support identification of novel anti-cancer therapeutics.

\section{INTRODUCTION}

Cancers can adapt to different environmental conditions, changing cellular morphologies and genetic characteristics in order to survive [1]. Cancer cells are responsible for activation of nearby stromal cells, including fibroblasts, endothelial cells and macrophages [2]. Activated stromal cells reorganize connective tissue structure and composition by releasing chemotactic cytokines and growth factors, and depositing extracellular matrix (ECM) components [3]. This enhances tumor growth and invasion and promotes chemotherapy resistance $[3,4]$. The aim of this work was to simulate the establishment of a tumor microenvironment between the osteosarcoma cell line, MG-63, and human fibroblasts (HFs) grown in co-culture. We showed that tumor and normal cell coexistence leads to morphological and molecular changes in both cell lines. Our analyses focused on some of the key factors involved in tumor induction and their roles in tumor angiogenesis, invasion, metastasis, and ECM composition.

TNF alpha

Tumor necrosis factor alpha (TNF- $\alpha$ ), a member of the TNF/TNFR cytokine superfamily, is assocated with chronic inflammation and the development of cancer. TNF- $\alpha$ expression within the tumor microenvironment promotes tumor cell invasion, migration and metastasis in several cancers. It partially activates the nuclear factor kappa-light-chain-enhancer of activated B cells (NF-kB) and the cytokine network in human cancer cells. It is also central to interactions between tumor cells and macrophages, promoting malignant cell invasion $[5,6]$. 
TNF- $\alpha$ is produced at low picogram levels in the tumor microenvironment, whether by tumor or stromal cells [6]. Increased serum TNF- $\alpha$ concentrations are observed in several cancers, despite tumor cells producing only small amounts of the molecule [6]. TNF- $\alpha$ may directly damage DNA, mediate tumor and stromal cell interactions, inhibit apoptosis, promote cell proliferation, and induce expression of tumor development-promoting molecules [6]. In established tumors, TNF- $\alpha$ contributes to the maintenance of a pro-inflammatory environment [6].

\section{IL-6}

Interleukin 6 (IL-6) is a multifunctional inflammatory cytokine involved in various biologic processes, including dysimmune diseases and cancers [7]. It orchestrates innate and adaptive immunity, mediates chronic inflammation and autoimmunity, and is a key cytokine linking chronic inflammation to cancer development [8]. Like TNF- $\alpha$, IL-6 facilitates tumor development by promoting the conversion of noncancer cells into tumor stem cells in vitro via Oct4 gene upregulation expression through IL-6R/JAK/STAT3 signaling [8]. Elevated IL-6 levels in human serum are associated with increased cancer risk. Thus, IL-6 is considered a prognostic marker and current anti-cancer therapies already target IL-6 activity [7].

\section{YKL-40}

YKL-40, also known as chitinase-3-like protein 1 , is a chitinase-like glycoprotein that lacks chitinase activity due to active site mutations. It interacts with glycosaminoglycans, including heparin and hyaluronan, and binds collagen types I-III. It suppresses E-cadherin, but increases matrix metalloproteinase-9 (MMP-9) expression and cell motility, which are each essential for tumor cell invasion [9]. YKL-40 purified from MG-63 cells induces fibroblast morphologic transformations near the tumor site, secretion of MMPs, and neovascularization [4], promoting cancer cell invasion and destruction of the stroma [10]. Serum YKL-40 levels are elevated in a variety of chronic inflammatory diseases, suggesting that its pathologic function involves ECM remodeling. Its expression is stimulated by cytokines, including IL-13, IL-6 and IL-1 $\beta[9]$.

\section{VEGF}

Vascular endothelial growth factor (VEGF) is a homodimeric glycoprotein that mediates tumor angiogenesis. VEGF is upregulated by oncogenes, growth factors, and hypoxia. VEGF also supports tumor growth by protecting tumor neovasculature against apoptosis, through induction of the anti-apoptotic factors, Bcl-2 and survivin [11]. Additionally, VEGF induces secretion and activation of enzymes involved in ECM degradation, such as plasminogen activator and MMP-1, allowing for further blood vessel development [12]. YKL-40 is a potent angiogenesis inducer, and has been investigated in several types of cancer. Faibish, et al. found that YKL40 up-regulates VEGF production in the glioblastoma cell line, U87, and demonstrated that monoclonal antibodies targeting YKL-40 activity are a promising strategy for treating advanced tumors [13].

\section{MMPs and MMP-9}

During tumor cell invasion, matrix metalloproteinases digest various ECM components, including proteoglycans, collagen, laminin, fibronectin, elastin, and vitronectin [14]. MMPs promote cancer cell invasion, migration, and metastasis [15]. The gelatinases, MMP-2 and MMP-9, degrade type IV collagen and gelatin, the two main ECM and basement membrane structural proteins. MMP-9 overexpression is associated with enhanced tumor cell invasion and overall aggression $[16,17]$.

\section{RESULTS}

\section{High resolution scanning electron microscopy (HR-SEM)}

HR-SEM images depicted a clearly identifiable morphology for the control samples from both human fibroblast (HF) and MG-63 cells. After 24 h, HFs alone exhibited a fibroblastic morphology with an elongated cell body (Figure 1A, arrow) and MG-63s were polygonal (Figure 1B, star). In co-cultures, HFs at $48 \mathrm{~h}$ appeared more disordered (Figure 1E, arrow). After quickly reaching confluence and forming clusters (Figure 1F, stars), MG-63 cells moved toward HFs, invading their space by $72 \mathrm{~h}$ (Figure 1H, stars) and overlapping them at $96 \mathrm{~h}$ (Figure 1I). Co-cultured MG-63 morphology changed at $96 \mathrm{~h}$ (Figure $1 \mathrm{~K}$ ) compared to controls (Figure $1 \mathrm{~K}-1 \mathrm{~L}$ ), showing some pseudopod protrusions and a pre-uropod region.

\section{Cell migration}

MG-63 cells cultured without HFs reached maximum migration levels of about $7 \%$ at $96 \mathrm{~h}$ (Figure 2A). When co-cultured with HFs, MG-63 migration gradually increased up to a maximum of $24 \%$ at $96 \mathrm{~h}$ (Figure 2A-2B).

\section{Western blot and densitometric analyses}

Densitometric analysis results showed lower TNF- $\alpha$ levels in co-cultured MG-63s than in HFs. In both cell lines, TNF- $\alpha$ bands reached maximum intensities after $96 \mathrm{~h}$ (Figure 3). IL-6 expression in MG-63s decreased after $24 \mathrm{~h}$ and remained stable. In HFs, IL-6 increased at $48 \mathrm{~h}$, then remained constant (Figure 4). YKL-40 expression 
in MG-63s increased until 96 h, while in HFs, YKL-40 was not expressed until $48 \mathrm{~h}$. Expression remained high in HFs at 72 and $96 \mathrm{~h}$ (Figure 5). MG-63s exhibited higher VEGF levels compared to HFs, with an increase at 72 and $96 \mathrm{~h}$. VEGF expression was low in HFs at 24 and $48 \mathrm{~h}$, reaching maximum levels at $72 \mathrm{~h}$ and decreasing again at $96 \mathrm{~h}$ (Figure 6). Low MMP-9 levels were detected in MG63 cells, with maximal levels at 72 and 96 h. Very high MMP-9 expression was observed in HFs after 24 and 48 $\mathrm{h}$, with a decrease after $72 \mathrm{~h}$ (Figure 7).

\section{DISCUSSION}

The tumor microenvironment actively contributes to cancer progression, and may direct genetic and epigenetic changes within cells involved. Recent studies showed that biochemical and physical signals could profoundly alter both tumor and nearby normal cells. New in vitro cell culture techniques allow for analysis of multiple physical and molecular culture parameters, including ECM pore size, fiber alignment, cell proliferation, and metastatic potential, clarifying how these components drive tumor progression. Stromal cells, such as fibroblasts or immune cells, can work in concert with cancer cells during tumor development to promote tumor progression via ECM modifications [18]. While stromal cells are not malignant, interactions with each other, the tumor microenvironment, and with cancer cells can induce phenotypic and functional changes [19]. Normal-malignant cell contact and crosstalk further drives the cancer phenotype $[19,20]$.

The aim of this work was to analyze the morphological and molecular changes that occour in cocultured human fibroblasts and osteosarcoma cells during a simulation of the tumor microenvironment. We observed direct stimulation of tumor cell growth, proliferation, and migration by growth factors and chemokines originating from both fibroblasts and cancer cells. A morphological HR-SEM analysis was performed on co-cultured MG-63 and HF cells grown in monolayers and on the same cells cultured alone. While fibroblasts seeded in co-culture seemed to lose their orderly organization patterns early, MG-63s exhibited characteristics of rapidly growing cells, reaching confluence faster than HFs. MG-63 cells formed clusters, intruding into HF space and almost overlapping them. Invasive malignant cells in co-culture appeared to change their shapes compared to controls, showing
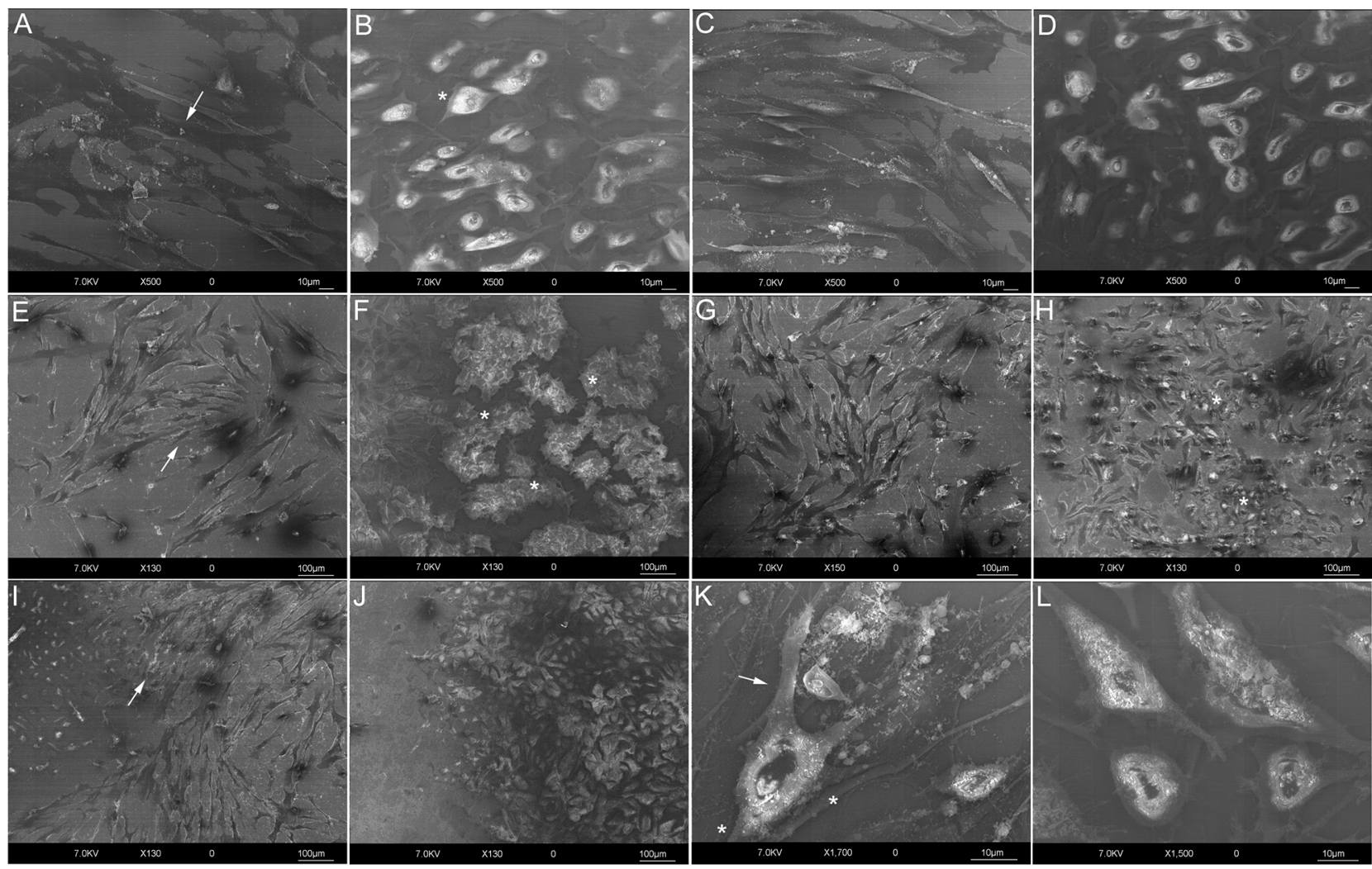

Figure 1: HR-SEM analyses. HF control at $24 \mathrm{~h}$ (Bar: $10 \mu \mathrm{m})$ A. Flat and elongated morphology (arrow). MG-63 control at $24 \mathrm{~h}$ (Bar: $10 \mu \mathrm{m})$ B. Polygonal morphology (star). HFs C. and MG-63s D. after 24 h of co-culture (Bar: $10 \mu \mathrm{m}$ ). HFs E. and MG-63s F. after 48 h of co-culture. Loss of fibroblast orientation (E., arrow); reduced MG-63 proliferation (Bar: $100 \mu \mathrm{m}$ ) and formation of clusters (F., stars). HFs G. and MG-63s H. after $72 \mathrm{~h}$ of co-culture (Bar: $100 \mu \mathrm{m})$. MG-63 invasion of HF space (H., stars). HFs I. and MG-63s J. after $96 \mathrm{~h}$ of coculture (Bar: $100 \mu \mathrm{m}$ ). Overlap of MG-63s with HFs (I., arrow). Detail of MG-63s at 96 h of co-culture K. Pseudopod protrusions (stars) and pre-uropod region (arrow) (Bar: $10 \mu \mathrm{m}$ ). Detail of MG-63 control at $96 \mathrm{~h}$ (Bar: $10 \mu \mathrm{m}) \mathbf{L}$. 
cytoskeletal polarization and protrusions. We believe that MG-63 cells in our study were in an early migration stage, showing relatively few specialized structures, like pseudopod protrusions opposite a "pre-uropod" region, as described by Friedl and Alexander [21, 22].

A cell migration assay in a trans-well system was performed to futher assess MG-63 cell migration in coculture with HFs. MG-63s showed little or no migration ( $7 \%$ at $96 \mathrm{~h}$ ) when cultured alone, but when co-cultured with HFs, MG-63 cell migration gradually increased throughout the experiment ( $24 \%$ at $96 \mathrm{~h})$. We hypothesized that MG-63s responded to HF-secreted paracrine signals and growth factors that acted as chemioattractants. Tumor cell dissemination is thought to occur in part via chemotaxis [23]. Directional migration toward a chemokine source is observed in vitro and in vivo for cells of the tumor microenvironment, leading to cancer cell invasion, inflammatory responses, and angiogenesis [23].
A

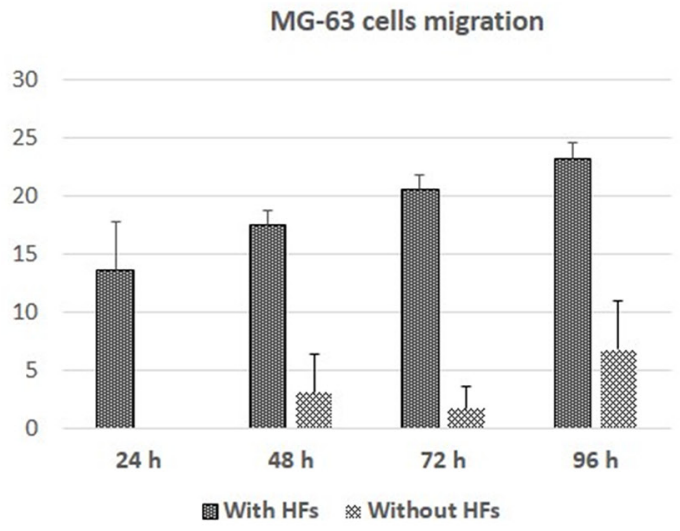

B

$24 \mathrm{~h}$

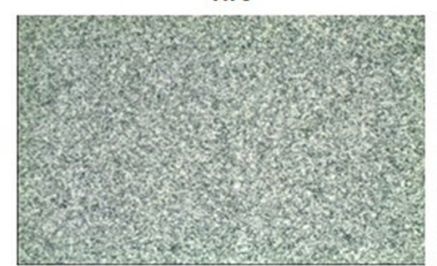

$48 \mathrm{~h}$

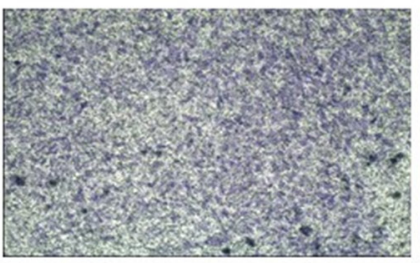

$72 \mathrm{~h}$

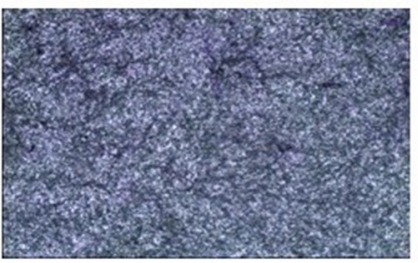

$96 \mathrm{~h}$

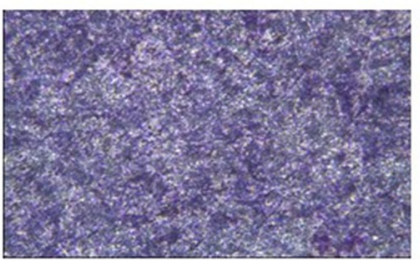

+ HFs
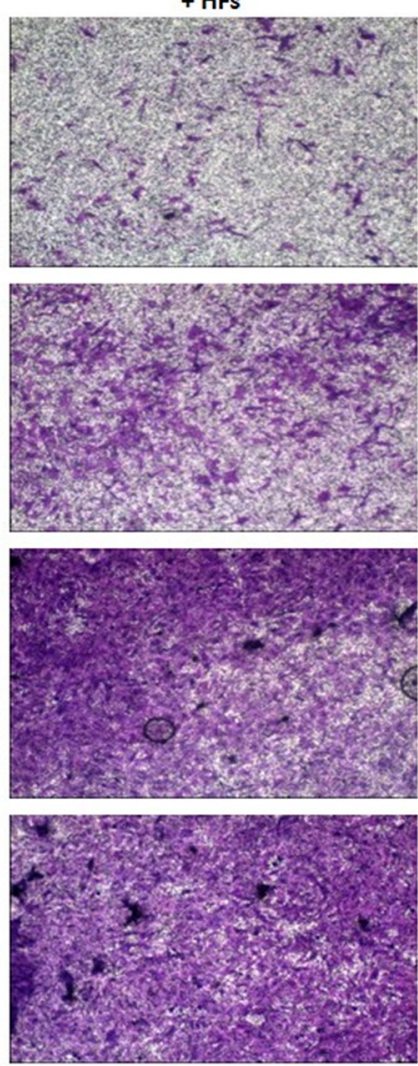

Figure 2: Cell migration assay. MG-63 cell migration (\%) A. * represents a difference between MG-63 cells cultured with and without HFs, $\mathrm{P}<0.05$. Giemsa staining of migrating MG-63 cells, with or without HFs $\mathbf{B}$.

TNF- $\alpha$ expression of MG-63s and HFs in co-culture

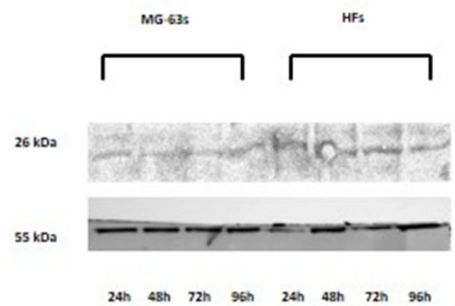

A

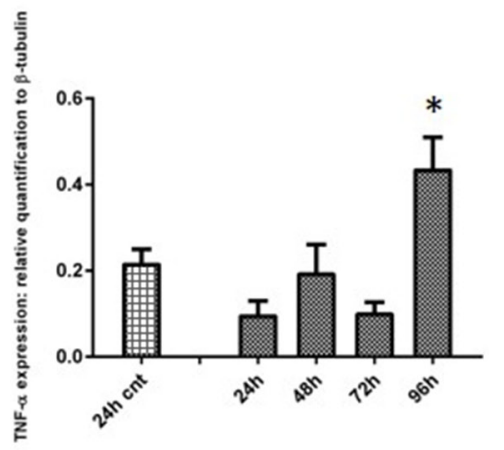

MG-63s in co-culture

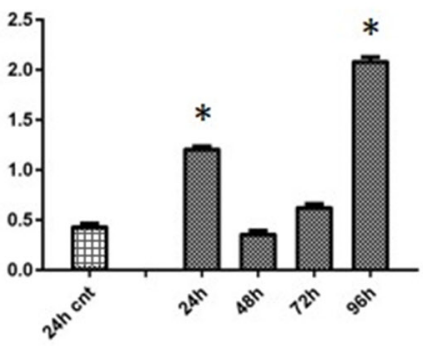

HFs in co-culture

Figure 3: Western blot for TNF- $\alpha$. Densitometric analysis A. TNF- $\alpha$ expression in co-cultured HFs and MG-63 cells B. * represents a difference between co-cultured cells and HF or MG-63 cells alone. $\mathrm{P}<0.05$. 
Some of the key factors involved in these processes were therefore analyzed in our study, including TNF- $\alpha$, IL-6, YKL-40, VEGF and MMP-9.

TNF- $\alpha$ and IL- 6 are central mediators of inflammation, and contribute to the maintenance of a pro-inflammatory environment. TNF- $\alpha$ is produced at low levels in the tumor microenvironment by tumor or stromal cells, or probably both [6], and perpetuates chronic inflammation and autoimmunity together with IL-6, a key cytokine linking chronic inflammation to cancer. Our western blotting results showed that TNF- $\alpha$ levels were lower in MG-63 cells than in HFs, and
IL-6 expression of MG-63s and HFs in co-culture

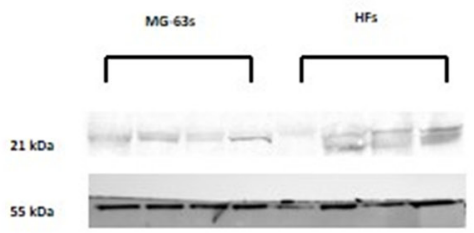

$24 \mathrm{~h} \quad 48 \mathrm{~h} \quad 72 \mathrm{~h} \quad 96 \mathrm{~h} \quad 24 \mathrm{~h} \quad 48 \mathrm{~h} \quad 72 \mathrm{~h} \quad 96 \mathrm{~h}$

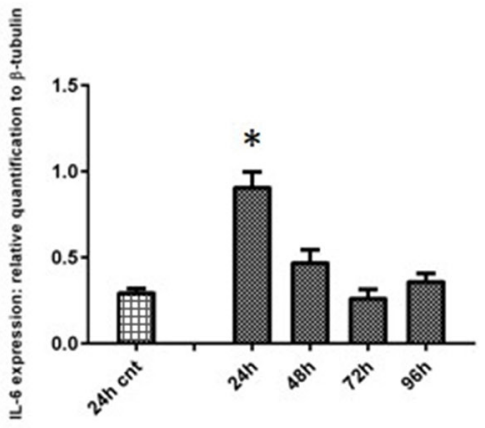

MG-63s in co-culture

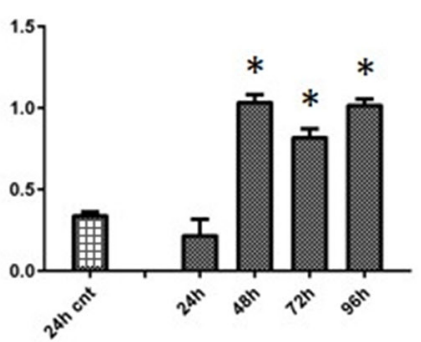

HFs in co-culture

Figure 4: Western blot for IL-6. Densitometric analysis A. IL-6 expression in co-cultured HFs and MG-63 cells B. * represents a difference between co-cultured cells and HF or MG-63 cells alone. $\mathrm{P}<0.05$.

YKL-40 expression of MG-63s and HFs in co-culture

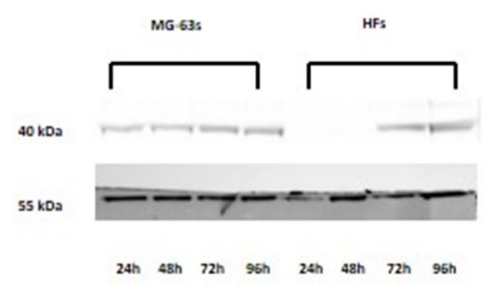

A

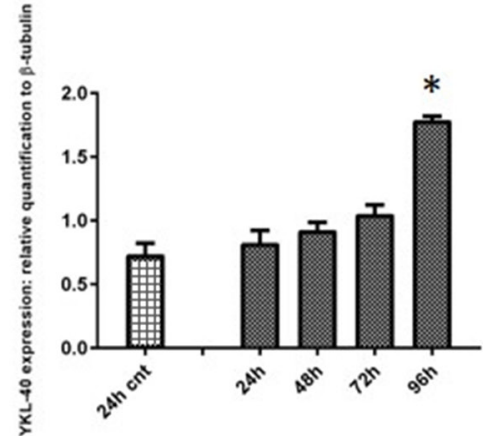

B

MG-63s in co-culture

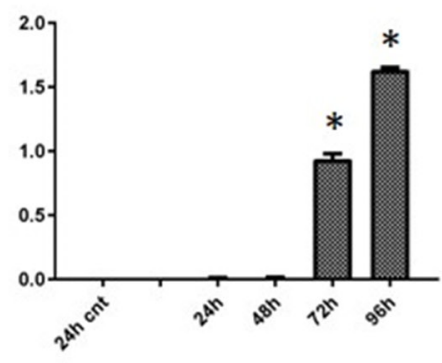

HFs in co-culture

Figure 5: Western blot for YKL-40. Densitometric analysis A. YKL-40 expression in co-cultured HFs and MG-63 cells B. * represents a difference between co-cultured cells and HF or MG-63 cells alone. $\mathrm{P}<0.05$.

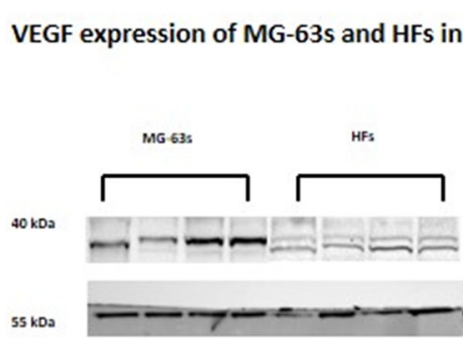

$24 \mathrm{~h} \quad 48 \mathrm{~h} \quad 72 \mathrm{~h} \quad 96 \mathrm{~h} \quad 24 \mathrm{~h} \quad 48 \mathrm{~h} \quad 72 \mathrm{~h} \quad 96 \mathrm{~h}$

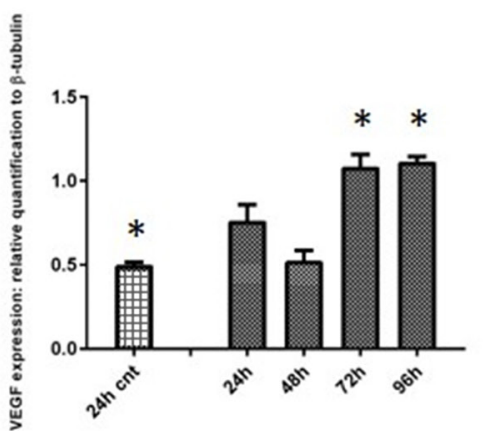

B

MG-63s in co-culture

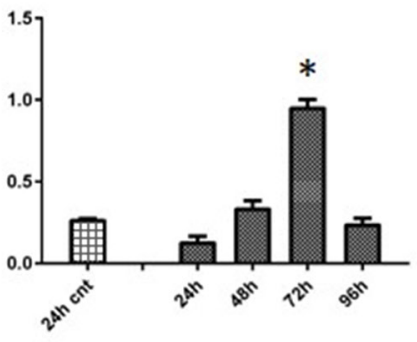

HFs in co-culture

Figure 6: Western blot for VEGF. Densitometric analysis A. VEGF expression in co-cultured HFs and MG-63 cells B. * represents a difference between co-cultured cells and HF or MG-63 cells alone. $\mathrm{P}<0.05$. 
densitometric analysis revealed that in both cell types, the signal increased through $96 \mathrm{~h}$ of co-culture. TNF- $\alpha$ expression induces rapid IL-6 activation in tissue affected by inflammation, which explains the increase in IL-6 expression in fibroblasts under co-culture conditions. HFs co-cultured with MG-63s showed increased IL-6 expression after $48 \mathrm{~h}$ [24].

As TNF- $\alpha$ and IL-6 are major regulators of acute inflammation, and IL-6 appears to promote YKL-40 expression [25], YKL-40 overexpression is associated with increased inflammation. YKL-40 is a glycoprotein normally expressed in vitro by MG-63 cells, potentially promoting the angiogenic switch and remodelling of the ECM, as hypothesized in our previous work [4]. YKL40 western blotting results confirmed our prior results: HFs that do not normally express YKL-40 in vitro begin to do so at $72 \mathrm{~h}$ when co-cultured with MG-63 cells, reaching the same maximum signal levels as $\mathrm{MG}-63 \mathrm{~s}$ at $96 \mathrm{~h}$. Moreover, band densitometric analyses showed that increasing YKL-40 expression was followed by VEGF overexpression, especially in MG-63s at 72 and $96 \mathrm{~h}$, confirming that YKL-40 promotes angiogenesis [13].

Finally, our results confirmed fibroblasts as the main source for MMPs in the tumor microenvironment. MMPs promote tumor cell invasion, migration, and metastasis [15], and originate from both tumor and benign cells, such as fibroblasts [26]. In part, this allows the socalled tumor-associated fibroblasts to stimulate tumor cell motility and invasion. MG-63 cells showed weak MMP-9 expression compared to HFs, which exhibited elevated protein levels after only $24 \mathrm{~h}$. This suggests that the tumor microenviroment modifies the stroma by upregulating the matrix metalloproteinases, which are responsible for ECM disruption, cell migration and tumor metastasis. The net balance between metalloproteinases and their specific endogenous tissue inhibitors (TIMPs) activities determines the proteolytic potential of tumors [27]. Decreases in TIMP levels are correlated with tumorigenesis, and in our model we presumed that MMP-
9 secreted by fibroblasts was not balanced by its inhibitor, TIMP-1. However, elevated TIMP-1 expression reportedly promoted cancer cell proliferation and invasion and correlated with progression and unfavourable prognosis in certain tumor types [27]. Analysis of TIMP-1 expression and activity by immunoblotting and zymography are still needed for a better understanding of our model, along with investigations of cellular responses to MMP/TIMP ratios.

Inhibition of MMP expression and/or activity may effectively prevent tumor invasion and metastasis. Recent strategies for blocking MMP gene transcription target extracellular factors, signal-transduction pathways, or nuclear factors that activate MMP gene expression [28]. The use of ribozymes or antisense constructs downregulates MMP production and/or TIMP1 expression [28]. Although there are many pathways toward blocking MMP production, none have been translated into the clinical, due in part to the diversity of MMP-regulating molecules, and the opposing effects of different factors on the expression of different MMPs [28]. These observations highlight the need for novel MMP inhibition strategies. A decade ago, the FDA approved Avastin as the first drug specifically targeting the tumor microenvironment in cancer [29]. Since this milestone, preclinical development and clinical testing of tumor microenvironment-targeting agents has increased remarkably, and several such agents are already standard treatments in patients with specific cancers [29]

As shown by the large number of chemotherapeutics approved by the FDA, strategies targeting the tumor vasculature also appear successful [29]. Furthermore, strategies inhibiting protumorigenic inflammatory responses are rapidly being developed with agents targeting pathways activated in tumor and stromal cells [29, 30]. Assessing new tumor microenvironmenttargeting agents in combination with radiotherapy and other chemotherapeutics may help avert tumor resistance to treatment. Additionally, because the pro- or antitumorigenic functions of the tumor microenvironment
MMP-9 expression of MG-63s and HFs in co-culture

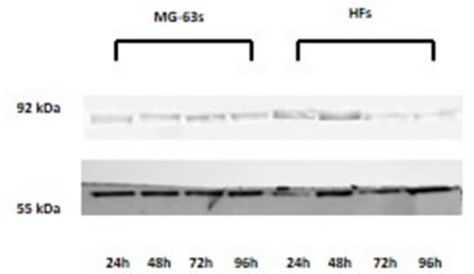

A

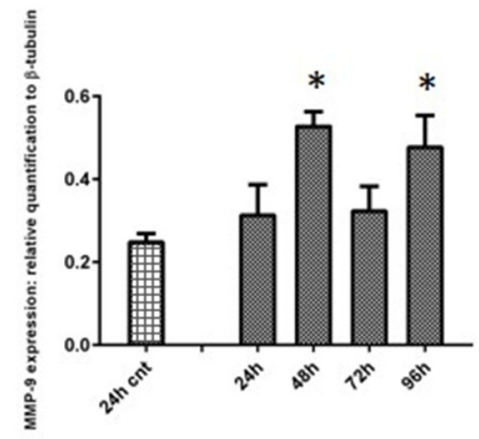

MG-63s in co-culture

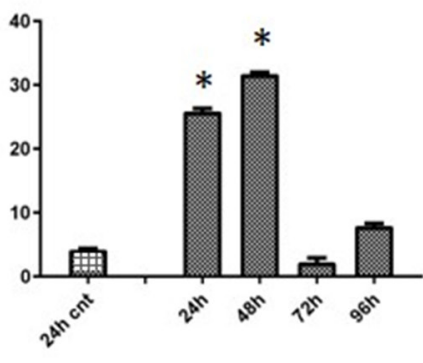

HFs in co-culture

Figure 7: Western blot for MMP-9. Densitometric analysis A. MMP-9 expression in co-cultured HFs and MG-63 cells B. * represents a difference between co-cultured cells and HF or MG-63 cells alone. $\mathrm{P}<0.05$. 
change during cancer progression, therapeutic agents must be assessed for effectiveness at various disease stages in specific cancers [29]. Reliable biomarkers that indicate the type of tumor stroma present are also needed for improved patient outcomes [29].

In conclusion, our results emphasize the microenvironment as an intrinsic aspect of the tumor itself. As the ECM differs between cancer types, in vitro systems should be adapted to simulate the native stromal environment. Experiments combining cancer and stromal cells are necessary to enhance understanding of such interactions during cancer cell invasion and metastasis [1, 20] and may lead to the identification of novel anti-cancer therapeutic strategies.

\section{MATERIALS AND METHODS}

\section{Primary human fibrobast cultures}

Human gingival tissues were collected from the midthird of the roots of teeth extracted for orthodontic reasons, with informed patient consent. After several washes in PBS, tissues were cut into small pieces and placed into culture dishes with $1 \mathrm{~mL}$ of Dulbecco's Modified Essential Medium (DMEM) (Invitrogen, Carlsbad, CA, USA) supplemented with $10 \%(\mathrm{v} / \mathrm{v})$ fetal bovine serum (FBS), penicillin (100 $\mathrm{mg} / \mathrm{mL})$, and streptomycin $(10 \mathrm{mg} / \mathrm{mL})$. Culture medium was changed twice per week. Subconfluent (70-80\%) HFs were detached from culture dishes using 0.05\% trypsin/ EDTA (Gibco, Grand Island, NE, USA), washed, and placed into T75 flasks. Cells were then cultured at $37^{\circ} \mathrm{C}$ in a humidified atmosphere with $5 \% \mathrm{CO}_{2}$. Cells from passages 3 to 10 were used for experiments.

\section{MG-63 cell culture}

The MG-63 osteosarcoma cell line was purchased from ATCC (Manassas, VA, USA; CRL-1427) and cultivated in DMEM supplemented with $10 \%$ (v/v) FBS, penicillin $(100 \mathrm{mg} / \mathrm{mL})$, and streptomycin $(10 \mathrm{mg} / \mathrm{mL})$. Cells were cultured in T25 flasks (Nunc, Waltham, MA, USA) in a humidified incubator at $37^{\circ} \mathrm{C}$ with $5 \% \mathrm{CO}_{2}$. For passaging, cells were detached with trypsin/EDTA and replated.

\section{HF and MG-63 cell co-cultures}

We performed a transwell co-culture, with $\mathrm{HF}$ and MG-63 cells seeded at $2.5 \times 10^{5}$ cells $/ \mathrm{mL}$ each. HFs were pre-cultured in 6-well plates, while MG-63 cells were seeded in 6-well transwell inserts (Transwell® permeable support, Corning Life Sciences). After 24 h, each support was put in the 6-well plates and co-cultured in DMEM supplemented with $10 \%(\mathrm{v} / \mathrm{v}) \mathrm{FBS}$, penicillin $(100 \mathrm{mg} /$ $\mathrm{mL})$, and streptomycin $(10 \mathrm{mg} / \mathrm{mL})$ for $24,48,72$, or 96 h. HFs and MG-63 cells were collected and analyzed at the same time points.

\section{HR-SEM analysis}

HF and MG-63 cells were seeded on sterile silica supports at $10 \times 10^{3}$ cells $/ \mathrm{ml}$ and co-cultured for $24,48,72$ or $96 \mathrm{~h}$ in petri dishes, in DMEM supplemented with $10 \%$ (v/v) FBS, penicillin (100 mg/mL), and streptomycin (10 $\mathrm{mg} / \mathrm{mL})$. The same cell types seeded separately on silica supports at the same density were used as control. At each collection point, silica supports were washed in sodium cacodylate buffer $0.15 \mathrm{M}$, fixed with $2.5 \%$ glutaraldehyde in $0.1 \mathrm{M}$ sodium cacodylate buffer (Sigma Aldrich, St. Louis, Missouri, USA) for $2 \mathrm{~h}$ at $4^{\circ} \mathrm{C}$, and post-fixed with $1 \% \mathrm{OsO}_{4}($ Società Italiana Chimici, Roma, Italia) in 0.1 $\mathrm{M}$ sodium cacodylate buffer for $1 \mathrm{~h}$ at room temperature. After several washes in $0.15 \mathrm{M}$ sodium cacodylate buffer, samples were dehydrated in increasingly concentrated alcohol solutions and then processed by critical point drying (030 Critical Point Dryer, Bal-Tec, Leica Microsystems GmbH, Wetzlar, Germany). Samples were metallized by a thin layer of platinum carbon (BalTec, Leica Microsystems $\mathrm{GmbH}$ ) and observed by HRSEM (JSM 890, Jeol Company, Tokyo, Japan) with an accelerated voltage of $10 \mathrm{kV}$ and $1 \times 10-11 \mathrm{~mA}$.

\section{Cell migration assay}

A transwell system (Transwell ${ }^{\circ}$ permeable support, Corning Life Sciences, membrane pore size: $3.0 \mu \mathrm{m}$ ) was used to analyze cell migration. MG-63 cells were harvested and suspended in serum-free DMEM at $2.5 \times 10^{5}$ cells $/ \mathrm{mL}$. The suspension was seeded into the upper chamber of the transwell system. HFs were harvested and suspended in serum-free DMEM at the same concentration and seeded in the bottom wells. As a control, MG-63s were suspended in DMEM serum free at the same concentration and seeded into the upper wells, while in the bottom wells $2 \mathrm{~mL}$ of DMEM supplemented with $10 \%(\mathrm{v} / \mathrm{v}) \mathrm{FBS}$, penicillin $(100 \mathrm{mg} / \mathrm{mL})$, and streptomycin $(10 \mathrm{mg} / \mathrm{mL})$ were added. The wells were incubated at $37^{\circ} \mathrm{C}$ for $24,48,72$ and $96 \mathrm{~h}$. At each collection point, medium was removed from the inserts, inserts were washed twice in PBS, and cells were fixed in formaldheyde $(3.7 \%$ in $\mathrm{PBS})$ for $2 \mathrm{~min}$ at room temperature. The formaldheyde was removed and cells were washed twice in PBS and permeabilized in 100\% methanol at room temperature for $20 \mathrm{~min}$. The methanol was removed and cells were washed twice in PBS and stained with Giemsa (Sigma Aldrich) at room temperature for $15 \mathrm{~min}$. Cells were then washed twice in PBS and non-migrated cells were removed with cotton swabs. Migrated cells were counted under a phase contrast microscope (Motic AE21, Seneco Srl, Milano, Italy). Membranes were photographed in triplicate and images were recorded using Visicam 3.0 and analyzed by VisiCam Image Analyzer software, version 6.1.3.3 (VWR International Srl, Milano, Italy). The stained area was quantifyed using ImageJ software (National Institutes of Health, Bethesda, MD) and expressed as a percentage (stained areas versus non-stained areas). 


\section{Western blotting and densitometric analysis}

At each experimental time point, cell pellets were lysed for 30 min using RIPA buffer (Invitrogen, Life Technologies, Monza Italy) supplemented with a protease inhibitor cocktail (Sigma Aldrich), $1 \mathrm{mM} \mathrm{PMSF}$ and $0.15 \% \beta$-mercaptoethanol (Fluka, Sigma Aldrich). Samples were centrifuged at $14,000 \mathrm{rpm}$ for $10 \mathrm{~min}$ at $4^{\circ} \mathrm{C}$, and total protein concentrations were measured using Bradford reagent (Sigma Aldrich). Twenty $\mu \mathrm{g}$ of total protein was resolved on $12 \%$ SDS-PAGE Bolt ${ }^{\circledR}$ Bis-Tris Plus MES gels (Invitrogen). Proteins were transferred to a nitrocellulose membrane (GE Healthcare Europe $\mathrm{GmbH}$ ), blocked with nonfat dry milk (Sigma Aldrich) for $30 \mathrm{~min}$ at room temperature, and immunolabeled with a primary antibody (anti-TNF- $\alpha$, IL-6, YKL- 40, VEGF, and MMP9 , with $\beta$-tubulin as endogenous control) diluted in TBS $\mathrm{pH} 7.5$ at $4^{\circ} \mathrm{C}$ overnight. Bands were visualized using an ECL Advanced TM Western blotting detection kit (GE Healthcare Europe $\mathrm{GmbH}$ ) and images were recorded with a Kodak digital image station (Eastman Kodak, Rochester, NY, USA). Band densitometric analysis was performed using Image $\mathbf{J}$ software, and band intensities were corrected for equal $\beta$-tubulin loading. Intensities are provided relative to the intensities of controls. Densitometry data are represented as means \pm standard deviations (SD) of three independent experiments.

\section{Statistical analysis}

Statistical analysis was carried out using GraphPad Prism 5.0 software (San Diego, CA) via analysis of variance (ANOVA) and the Dunnet's multiple comparison test. $\mathrm{P}<0.05$ was considered statistically significant.

\section{Abbreviations}

HFs: Human fibroblasts; ECM: Extracellular matrix; TNF alpha: Tumor necrosis factor alpha; IL-6: Interleukin 6; YKL-40: Human cartilage glycoprotein-39; MMP-9: Matrix metalloprotease 9; MMPs: Matrix metalloproteinases; VEGF: Vascular Endothelial Growth Factor.

\section{ACKNOWLEDGMENTS}

This study was supported by Fondazione Del Monte di Bologna e Ravenna, Italy.

\section{CONFLICTS OF INTEREST}

The authors declare no conflicts of interest.

\section{FUNDING}

This study was funded by the Italian Ministry of Research and Technology (MURST).

\section{Author contributions}

Viviana Salvatore and Mirella Falconi participated in the concept and design of the study. Viviana Salvatore performed experiments, analyzed data, prepared figures and wrote the manuscript. Stefano Focaroli, Gabriella Teti, Maria Carla Mazzotti and Antonio Mazzotti carried out the acquisition of data and statistical analysis. Mirella Falconi has given final approval of the version to be published. All the authors have read and approved the final manuscript.

\section{REFERENCES}

1. Clark AG, Vignjevic DM. Modes of cancer cell invasion and the role of the microenvironment. Curr Opin Cell Biol. 2015; doi: 10.1016/j.ceb.2015.06.004.

2. Friedl P, Alexander S. Cancer invasion and the microenvironment: plasticity and reciprocity. Cell, 2011; doi: 10.1016/j.cell.2011.11.016.

3. Egeblad M, Rasch MG, Weaver VM. Dynamic interplay between the collagen scaffold and tumor evolution. Curr Opin Cell Biol. 2010; doi: 10.1016/j.ceb.2010.08.015.

4. Salvatore V, Focaroli S, Teti G, Falconi M. Changes in the gene expression of co-cultured human fibroblast cells and osteosarcoma cells: the role of microenvironment. Oncotarget. 2015; 6:28988-98. doi: 10.18632/oncotarget.4902

5. Mocellin S, Nitti D. TNF and cancer: the two sides of the coin. Front Biosci. 2008; 2774-2783.

6. Balkwill F. Tumour necrosis factor and cancer. Nat Rev Cancer. 2009 doi: 10.1038/nrc2628.

7. Rossi JF, Lu ZY, Jourdan M, Klein B. Interleukin-6 as a therapeutic target. Clin Cancer Res. 2015; doi: 10.1158/1078-0432.CCR-14-2291.

8. Landskron G, De la Fuente M, Thuwajit P, Thuwajit C, Hermoso MA. Chronic Inflammation and Cytokines in the Tumor Microenvironment. Journal of Immunology Research. 2014 doi: 10.1155/2014/149185.

9. Libreros S, Garcia-Areas R, Iragavarapu-Charyulu V. CHI3L1 plays a role in cancer through enhanced production of pro-inflammatory/pro-tumorigenic and angiogenic factors. Immunol Res. 2013; doi: 10.1007/s12026-013-8459-y.

10. Rønnov-Jessen L, Petersen OW, Bissell MJ. Cellular changes involved in conversion of normal to malignant breast: importance of the stromal reaction. Physiol Rev. 1996; 76:69-125.

11. Carmeliet P. VEGF as a Key Mediator of Angiogenesis in Cancer. Oncology 2005; doi: 10.1159/000088478.

12. Unemori EN, Ferrara N, Bauer EA, Amento EP: Vascular endothelial growth factor induces interstitial collagenase expression in human endothelial cells. J Cell Physiol 1992; 153:557-562.

13. Faibish M, Francescone R, Bentley B, Yan W, Shao R. A YKL-40-neutralizing antibody blocks tumor angiogenesis and progression: a potential therapeutic agent in cancers. Mol. Cancer Ther. 2011;10:742-751. 
14. Roomi MW, Monterrey JC, Kalinovsky T, Rath M, Niedzwiecki A. Patterns of MMP-2 and MMP-9 expression in human cancer cell lines. Oncology Reports 2009; 21: 1323-1333.

15. Stetler-Stevenson WG: The role of matrix metalloproteinases in tumor invasion, metastasis and angiogenesis. Surg Oncol Clin N Am 2001; 10: 383-392.

16. Di Nezza LA, Misajon A, Zhang J, Jobling T, Quinn MA, Ostör AG, Nie G, Lopata A, Salamonsen LA: Presence of active gelatinases in endometrial carcinoma and correlation of matrix metalloproteinase expression with increasing tumor grade and invasion. Cancer 2002; 94: 1466-1475.

17. Schmalfeldt B, Prechtel D, Härting K, Späthe K, Rutke S, Konil E, Fridman R, Berger U, Schmitt M, Kuhn W, Lengyel E: Increased expression of matrix metalloproteinases (MMP)-2, MMP-9, and the urokinasetype plasminogen activator is associated with progression from benigh to advanced ovarian cancer. Clin Cancer Res. 2001; 7: 2396-2404.

18. Spill F, Reynolds DS, Kamm RD, Zaman MH. Impact of the physical microenvironment on tumor progression and metastasis. Curr Opin Biotechnol. 2016; doi: 10.1016/j. copbio.2016.02.007.

19. H Li, X Fan, JM Houghton. Tumor microenvironment: the role of the tumor stroma in cancer. Journal of Cellular Biochemistry, 2007; 101:805-815.

20. Clark AG, Vignjevic DM. Modes of cancer cell invasion and the role of the microenvironment. Curr Opin Cell Biol. 2015; doi: 10.1016/j.ceb.2015.06.004.

21. Friedl P, Alexander S. Cancer Invasion and the Microenvironment: Plasticity and Reciprocity. Cell. 2011; doi: 10.1016/j.cell.2011.11.016
22. Poincloux R, Collina O, Lizárragaa F, Romaoa M, Debraye M, Piela M, Chavrier P. Contractility of the cell rear drives invasion of breast tumor cells in 3D Matrigel. PNAS. 2011; doi: 10.1073/pnas. 1010396108

23. Roussos ET, Condeelis JS, Patsialou A. Chemotaxis in cancer. Nat Rev Cancer. 2011; doi: 10.1038/nrc3078.

24. Lippitz BE. Cytokine patterns in patients with cancer: a systematic review. Lancet Oncol. 2013; doi: 10.1016/ S1470-2045(12)70582-X.

25. Nielsen AR, Plomgaard P, Krabbe KS, Johansen JS, Pedersen BK. IL-6, but not TNF-a, increases plasma YKL40 in human subjects. Cytokine. 2011:152-155.

26. Cirri P, Chiarugi P. Cancer associated fibroblasts: the dark side of the coin. Am J Cancer Res. 2011;1:482-97.

27. Birgisson H, Nielsen HJ, Christensen IJ, Glimelius B, Brünner N. Preoperative plasma TIMP-1 is an independent prognostic indicator in patients with primary colorectal cancer: a prospective validation study. Eur J Cancer. 2010; doi: 10.1016/j.ejca.2010.06.009.

28. Overall CM, López-Otín C. Strategies for MMP inhibition in cancer: innovations for the post-trial era. Nature Reviews Cancer 2002. 2: 657-672.

29. Hua F, A. DeClerck Y. Targeting the Tumor Microenvironment: From Understanding Pathways to Effective Clinical Trials. Cancer Res. 2013; doi: 10.1158/0008-5472.CAN-13-0661.

30. Mercier I, Camacho J, Titchen K, Gonzales DM, Quann $\mathrm{K}$, Bryant KG, Molchansky A, Milliman JN, WhitakerMenezes D, Sotgia F, Jasmin JF, Schwarting R, Pestell RG, Blagosklonny MV, Lisanti MP. Caveolin-1 and accelerated host aging in the breast tumor microenvironment: chemoprevention with rapamycin, an mTOR inhibitor and anti-aging drug. Am J Pathol. 2012; 181: 278-93. 